This item was submitted to Loughborough's Research Repository by the author.

Items in Figshare are protected by copyright, with all rights reserved, unless otherwise indicated.

\title{
3D printed microfluidic device with integrated optical sensing for particle analysis
}

PLEASE CITE THE PUBLISHED VERSION

https://doi.org/10.1016/j.snb.2017.10.041

PUBLISHER

(C) Elsevier

VERSION

AM (Accepted Manuscript)

\section{PUBLISHER STATEMENT}

This work is made available according to the conditions of the Creative Commons Attribution-NonCommercialNoDerivatives 4.0 International (CC BY-NC-ND 4.0) licence. Full details of this licence are available at: https://creativecommons.org/licenses/by-nc-nd/4.0/

\section{LICENCE}

CC BY-NC-ND 4.0

\section{REPOSITORY RECORD}

Hampson, Sarah, William Rowe, Steven Christie, and Mark Platt. 2019. "3D Printed Microfluidic Device with Integrated Optical Sensing for Particle Analysis”. figshare. https://hdl.handle.net/2134/27302. 
3D Printed Microfluidic Device With Integrated Optical Sensing for Particle Analysis.

S. M. Hampson ${ }^{1}$, W. Rowe ${ }^{1}$, S.D.R. Christie ${ }^{1}$ and M. Platt ${ }^{1 *}$

1.Department of Chemistry, Loughborough University, Loughborough, Leicestershire, LE11 3TU, UK.

*m.platt@lboro.ac.uk,

\begin{abstract}
:
The integration of particle counters within lab-on-chip (LOC) microfluidic devices creates a range of valuable tools for healthcare such as cell counting, and synthesis applications e.g. materials fabrication. Avoidance of long and/ or complex fabrication processes can aid the uptake of these devices, specially within resource-poor societies. We present an additively manufactured microfluidic particle counter. The device features a hydrodynamic focusing chamber to stream the particles past embedded optical fibres for their detection. The intensity of occluded light through the fibre was found to be related to the size of the particles, allowing particles of different sizes to be identified. The signal-to-noise ratio and reproducibility of the particle analysis was optimised to three objectives (pulse magnitude, uniformity and periodicity) via the use of a genetic algorithm (GA). Once optimised the device was able to count particles upto $5.5 \times 10^{4}$ particles $\mathrm{ml}^{-1}$, and size particles in a mixture.
\end{abstract}

Key Words: 3D-Printed, Microfluidic, Additive Manufacturing, Particle analysis, Genetic Algorithm 


\section{Introduction}

Modern society is dependent upon the accurate and rapid characterisation of materials, from biological particles (cells, virus or bacteria) to inorganic materials. There exists a demand for portable particle/cell detection devices with applications in health diagnostics ${ }^{1}$, quality control (QC) systems for pharmaceutical manufacture ${ }^{2}$, and environmental applications ${ }^{3}$. Microfluidic sample handling and detection systems are one solution to this, owing to their unique fluid regimes and high surface-to-volume ratios that allow precise analyte manipulation and controlled, reproducible conditions ${ }^{4}$.

There exist many examples of microfluidic platforms that have integrated particle/cell detection ${ }^{5,6}$, including resistive pulse sensing ${ }^{7-10}$, particle image velocimetry ${ }^{11}$, magnetoresistive sensing ${ }^{12}$ and fluorescence-based counting ${ }^{13}$. However, these can involve complex fabrication, large, expensive external support systems, or sample pre-treatment steps such as labelling. A popular approach to this problem is flow cytometry (FC) a technique used in the clinical, healthcare and biotechnology sciences for the counting and analysis of cells (or other entities such as bacteria and particles), usually between 0.5 and $30 \mu \mathrm{m}$ in diameter ${ }^{14}$. Its principles are covered in detail elsewhere ${ }^{14-16}$, but primarily consist of two key steps: particle alignment into single file via hydrodynamic flow focusing, followed by sample analysis by a laser detection system or impedance ${ }^{15}$. Microfluidic flow cytometers are already predicted to be a means of innovation in the point-of-care (POC) and QC fields ${ }^{17-19}$, and there exists many reviews about this growing field $17,18,20-24$. Their ability for high throughput individual particle analysis makes them, and their components a gold standard technology. The resolution in particle size and throughput for microflow cytometers has been aided by developments within optics systems, namely, elements such as lenses and beam shaping ${ }^{17}$, and devices have chiefly utilised scattering and fluorescence detection ${ }^{25-27}$. Watts et al. detected 1,2 and $5 \mu \mathrm{m}$ beads $^{25}$, Fan et al. detected $10 \mu \mathrm{m}$ fluorescent beads ${ }^{26}$, and Etcheverry et al. detected 2 , 10,15 , and $24 \mu \mathrm{m}$ beads ${ }^{27}$, all with sample throughputs of thousands per second and all utilising laser systems. Watts et al. also used integrated, on-chip lenses ${ }^{25}$, Fan et al. used an intricate external lens system ${ }^{26}$, and Etcheverry et al. used filters, dichroic mirrors and multiple PMTs ${ }^{26}$.

A common fabrication process for such systems is via soft lithography, a multi-step process involving master mould creation, formation of device layers, assembly and bonding ${ }^{28}$. 3D printing has been suggested as an alternative approach to microfluidic fabrication, mainly due to its ability to build a complete part from an STL file with no intermediate steps, thus minimising labour, time and $\operatorname{costs}^{29}$. In 2014 Folch et al. estimated that the price to have a microfluidic device built by stereolithography (SLA) via a printing service was roughly equal to the price of SU-8 master mould fabrication for the same device ${ }^{30}$. Furthermore, in the last few months a number of major 3D printing companies have unveiled continuous, automated AM systems designed for rapid, low-cost mass production: the Demonstrator FDM system offered by Stratasys, and the Form Cell and Figure 4 SLA systems developed by Formlabs and 3D Systems, respectively. Additionally, due to the direct formation from a digital file format, design iterations (an integral part of the prototyping process) are greatly accelerated, and the removal of fabrication by layers allows topological design freedom. These factors make 3D printing a means for design innovation in the field of microfluidics where over the last 3 years the use of 3D printing for microfluidics and lab-on-a-chip fabrication has exploded ${ }^{31-42}$. An overview of microfluidic technologies made by each type of additive manufacturing is given by Folch $^{32}$, but SLA has proven to be the most popular for this field, due to its superior resolution ${ }^{30}$. SLA is a vat photopolymerisation process, involving the curing by photopolymerisation of liquid polymers inside a vat, via use of a laser to solidify material layer-by-layer ${ }^{42,43}$. 
Integration of sensing capabilities into 3D printed microfluidic devices is currently dominated by electrochemical systems, commonly using integrated fittings containing embedded electrodes ${ }^{44-47}$, or similar configurations ${ }^{48-50}$. Other systems have included an integrated chemoresistive gas sensor for VOC detection ${ }^{51}$ and a light-addressable potentiometric sensor (LAPS) ${ }^{52}$, or the integration of optical detection utilising embedded optical fibres ${ }^{53,54}$. Regardless of manufacturing, process sensitivity and reproducibility are requisites of any quantitative technology. Variations in manufacturing processes, even if printed from an identical CAD file, on different printers across the globe, may lead to different levels of sensitivity. To this end, it is often necessary to optimise a series of experimental parameters of the new system to maximise the output.

In this paper we present an optofluidic particle characteriser fabricated by SLA. It is the first additively manufactured microfluidic system to have integrated particle detection, via use of embedded optical fibres for particle-by-particle counting and analysis. Previous printed UVIVIS microfluidic systems have monitored liquid analytes ${ }^{53,54}$, or carried out spectroscopy externally, such as in non-integrated flow cells ${ }^{55}$. The device presented herein incorporates hydrodynamic focusing, took approximately 5 hours to build, and could be reused dozens of times. The embedded fibres were first used to monitor a focused core stream diameter via measurement of the absorption of a dye in the sample solution. Subsequent experiments incorporated $30 \mu \mathrm{m}$ diameter polystyrene beads into the sample stream and the run conditions were then optimised by a genetic algorithm. Screening of five experimental conditions (fibre core diameter, particle concentration, inner/sheath applied pressure ratio, overall applied pressure and the presence of a dye in the sample core stream) was carried out relative to three objectives: pulse magnitude (maximise), pulse magnitude variance (minimise) and pulse frequency variance (minimise). The algorithm was run for three generations, identifying a suitable set of conditions that met these objectives, which was then used to demonstrate particle counting across a range of concentrations over 2 orders of magnitude, and then differentiate $10 \mu \mathrm{m}$ and $30 \mu \mathrm{m}$ beads in a mixture.

\section{Experimental}

Materials and methods

Device fabrication Siemens NX 8.5 software was used to draft the device's computer-aided design (CAD) file (Fig. S1, ESI†) and export to .STL format. The microfluidic device was fabricated in Accura ${ }^{\circledR} 60$ polymer on a Viper si2 SLA printer (both 3D Systems) in high resolution (HR) build mode. This pre-set mode utilises a beam diameter of $75 \mu \mathrm{m}$ and layer thickness of $50 \mu \mathrm{m}$. Post printing, the device was rinsed with isopropyl alcohol to remove any uncured resin. The device was printed in three different build directions (Fig. S2, ESI $\dagger$ ) to ascertain the effects on channel and fluid flow quality. Multimode optical fibre ( $0.22 \mathrm{NA}$, silica core, glass clad, acrylate coating, core diameter $105 \mu \mathrm{m} \pm 2 \%$, cladding diameter $125 \mu \mathrm{m} \pm 1 \mu \mathrm{m}$, coating diameter $250 \mu \mathrm{m} \pm 4 \%$, wavelength range 250-1200 nm, obtained from Thorlabs) was used to transmit light. Optical alignment was achieved via the inclusion of grooves to house optical fibres in the CAD file. Before manual embedding, optical fibres were polished by hand using a series of lapping films. Device microchannels and grooves were printed open, and optical fibres were pressed into the grooves using a rubber nub and aligned with the channel walls by hand, under a microscope. Subsequent device sealing was done with TESA 4965 double-sided tape (obtained from 3M) and LEXAN ${ }^{\text {TM }}$ 8010PC polycarbonate film (obtained from SABIC, $250 \mu \mathrm{m}$ thickness), applied by hand and flattened with a seam roller. 
Microfluidic flows were driven by Mitos P-Pump Basic pressure-driven pumps (Dolomite) and fed into the device via standard HPLC fittings in printed threads. Light was supplied and collected using a MINI-D2T Miniature Deuterium Tungsten Light Source and an S2000 Miniature Fibre Optic Spectrometer, respectively (both Ocean Optics), the latter controlled via SpectraSuite software (Ocean Optics). Light intensities were recorded at a single wavelength as raw counts at an integration time of $3 \mathrm{~ms}$ and a $15 \mathrm{~ms}$ acquisition period. Device performance was tested and optimised using methylene blue $(1.0 \mathrm{M})$ and solochrome red $\mathrm{B}(1.0 \mathrm{M})$ aqueous dye solutions, and two sizes of calibration beads $\left(10 \mu \mathrm{m}\right.$ diameter, Fluoresbrite ${ }^{\circledR}$ Yellow Green carboxylated microspheres, polystyrene, concentration given by manufacturer as $4.55 \times 107$ particles $\mathrm{ml}^{-1}$, Polysciences, (18142-2) and $30 \mu \mathrm{m}$ diameter, polystyrene, Sigma Aldrich, (84135) in aqueous suspensions. Footage of particle focusing was recorded on a Meros high-speed digital microscope (Dolomite). Collected pulse spectra were smoothed of noise via Origin software (OriginLabs), using a 220-point (for bead mixture study) or 50-point (for all other runs), non-weighted, adjacentaveraging smooth function.

Fluid core stream analysis: Absorption of methylene blue $(1.0 \mathrm{M})$ core streams was measured by monitoring of light intensity at $665 \mathrm{~nm}$ and the core stream width was then calculated via rearrangement of the Beer-Lambert law. Visual imaging of the core stream widths using a microscope was done using methylene blue $(1.0 \mathrm{M})$ via photographing with an Optiphot-2 microscope (obtained from Nikon and controlled with a DS-L1 Digital Sight Camera Control Unit, also Nikon) followed by sizing via AxioVision software (Zeiss).

Peak analysis: Particle pulse spectra were taken by monitoring of collected light at $650 \mathrm{~nm}$. Peak calling was based on a moving window background of 50 data points (with the exception of a study of differently-sized beads, where an overall average baseline was used). Peaks were determined to be significant if the light intensity drop exceeded 5 standard deviations (SDs) from the mean of this window or baseline. This threshold was reduced to 3 SDs when determining the dose response at an optimised set of conditions (based on a great number of datasets), as well as when analysing a bead mixture.

Evolutionary optimisation: Evolutionary optimisation was performed over 3 generations, where the number of experiment runs were 20, 16 and 19 within each generation (Table S1, ESI†). The first generation comprised of conditions randomly generated within experimentally defined boundaries: Two intrinsic a) 50 or $105 \mu \mathrm{m}$ optical fibre core diameters, b) the use of water or methylene blue solution (1.0 M, aqueous) for the bead suspension solvent, and three continuous variables: c) particle concentrations ranging between $5.0 \times 10^{3}$ and $2.5 \times 10^{5} \mathrm{ml}^{-1}$, d) 1-3.5 inner flow/ sheath flow applied pressure ratios, e) 100-375 mbar total applied pressure (in the form of total pressures used across the three pumps). Fig. S3 shows a flowchart illustrating the GA process. PESA-II was used to select the best experimental conditions from the previous generation, and then modify these variables to produce a new generation. PESA-II was implemented as described as in Jarvis et al ${ }^{56}$. The algorithm was adapted such that every set of new conditions were modifications of the previous generation, with the exception of a group of the best sets of conditions (selected from the Pareto front) from the previous generation. The four best sets of conditions remained unchanged, serving as both controls and a metric of progress. Variables were mutated with a probability of 2/L (where $L$ represents the total number of variables). When mutation was applied, each of the variables were modified (increased or decreased) by up to $20 \%$ (a uniform random number between $0-20 \%$ of the original value). Tube diameter and dye were encoded as a decimal $x$, where for example, if $x>0.5$, dye was added to the solution, and if $x \leqslant 0.5$ dye was omitted. $60 \%$ of the experiments in the new 
generation were generated through uniform crossover, i.e. combining variables from the best solutions.

Bead counting runs: 7 different concentrations of $30 \mu \mathrm{m}$ bead were used ranging from $1 \times 10^{3}$ to 1.5 $\times 10^{5}$ beads $\mathrm{ml}-1$, run at the optimum conditions identified above (inner flow/sheath flow applied pressure ratio of 2.63 and total applied pressure of 207 mbar).

Bead size run: $A$ 1:1 aqueous mixture of 30 and $10 \mu \mathrm{m}$ diameter beads was made, with both concentrations being $1.25 \times 10^{4}$ beads $\mathrm{ml}^{-1}$. A $30 \mu \mathrm{m}$ diameter bead suspension (aqueous, $2.5 \times 10^{4}$ beads $\mathrm{ml}^{-1}$ ) and a $10 \mu \mathrm{m}$ diameter bead suspension (aqueous, $2.5 \times 104$ beads $\mathrm{ml}^{-1}$ ) were also made, and all were run at found optimum conditions.

\section{Results and discussion}

The particle counter was comprised of three basic parts: 1) three inlets and one outlet, 2) a hydrodynamic flow focusing junction and 3) embedded optical fibres (Fig. 1a). A photograph of the printed device is shown in Fig. 1b, and its CAD file for comparison in Fig. S1. To ensure that the focused stream of particles passed through the sensing region between the two optical fibres, the channel dimensions were made as close to the limit of resolution for the printer, whilst still maintaining a reproducible product. Microchannel dimensions were $250 \mu \mathrm{m}$ depth, $250 \mu \mathrm{m}$ width for the sample flow inlet, $400 \mu \mathrm{m}$ width for the sheath flow inlets, and $400 \mu \mathrm{m}$ width for the outlet channel. Smaller channels were tested but were prone to blockages or a poor quality final product (irregular channels, data not shown). Channels were printed on the surface of the device as this has been previously shown to improve printing resolution in SLA-made microfluidic devices ${ }^{53,57}$. Channels were also printed square to minimise the staircase effect, and to aid the alignment of the optical fibres with the microchannel walls.

The optical fibre groove junction for particle detection is shown in Fig. 1c. The fibres were embedded and aligned in the device as described in the experimental. A prototype device design was printed in three different print orientations (Fig. S2, ESIt) to test the resolution and reproducibility of the fabrication process. Two dye solutions were used to ascertain the ability of each chip to produce laminar flow and a focused, neatly centralised core stream. Examples of this are shown in Fig. 1d. The X-direction device suffered from an obstructing central support structure artefact, added automatically post design by the CAD software shown by the arrow in Fig 1.d. In addition, the rough channel wall on one side (due to laser over-curing of the channel ceiling) causing asymmetrical core stream focusing. Whilst both the Z-direction and $Y$-direction builds produced smooth microchannel walls, we opted for the $\mathrm{Y}$-direction build as it consistently gave the most reproducible at maintaining the small channel features (especially the end of the sample channel, as seen in Fig. 1d)- the Zdirection build has a narrowed section). It was also found that the Y-direction could achieve the thinnest stable dye stream for a 200 mbar inner flow applied pressure (Fig. S4, ESI†) of the three. Due to these factors, the $\mathrm{Y}$-direction was utilised to print the device used for the following dye and particles studies in this paper.

Hydrodynamic focusing of dye and particle streams

The processes of hydrodynamic focusing are illustrated in Fig. 1a. A sample suspension flow is introduced to the flow focusing chamber, whereupon it undergoes narrowing by two flanking sheath flow streams. This allows individual beads/samples to be characterised as they pass through an optical interrogation region between the two optical fibres. The hypothesis was that the passage of a bead/cell would cause a drop in light intensity, and through the continuous monitoring of light output over time the number of pulses would be proportional to the concentration of the beads. The 
stability of hydrodynamic focusing and the sensitivity of the optical detection system was first tested by focusing a core stream composed of methylene blue solution. Fig. 2a shows photographs of focused dyed core streams of varying thicknesses produced by decreasing the inner flow/sheath flow applied pressure ratio (left-to-right). As the sheath stream flowrates are increased relative to the inner stream flow rate, the inner stream is constricted and accelerated into a fast, narrow core stream. Fig. $2 b$ shows a linear plot of the measured absorbance at $665 \mathrm{~nm}$ (aqueous methylene blue $\left.\lambda_{\max }\right)$ for different thicknesses of dye core streams. Highly stable, uniform laminar flow was observed for all but the very widest streams (270, 319 and $384 \mu \mathrm{m}$ widths), which had some fluctuation in their $\lambda_{\max }$ absorption. Streams could be focused and detected down to approximately $3 \mu \mathrm{m}$ thickness, which was determined by measuring of stream photographs with image sizing software. Fig. $2 c$ shows the stability over time in absorbance for different core stream thicknesses.

\section{Optimisation of particle flow cytometry}

Having verified that the core stream width could be controlled and varied upon demand, the system's experimental conditions were optimised for the counting and analysis of $30 \mu \mathrm{m}$ diameter polystyrene beads. Two videos of examples of particle flow are found in the SI. Three experimental parameters were chosen to be optimised by the GA: 1) Signal reproducibility (variation in light intensity drop caused by a particle traversing the fibre optic junction). An ideal system would have little variation in pulse magnitude for uniformly sized particles. Thus the GA was directed to minimise this value which was inferred to result from a steady and stable flow stream of particles through the fibre optic path. 2) Signal periodicity (variation in time between pulses). To maximise the number of particles that could be counted per unit time the GA was directed to minimise this value. 3) The signal-tonoise ratio (pulse magnitude). The GA was directed to maximise this value.

Closed loop evolution is a method that iteratively utilizes the Darwinian processes of mutation (modification) and selection in the lab, to traverse the potential search space ${ }^{58}$. While the technique has been in existence for over fifty years, it has gained renewed popularity in the development of airfoils $^{59}$, aptamers ${ }^{60}$ and adaptive robotics ${ }^{61}$. Evolutionary multiobjective optimisation (EMO) extends the process to facilitate the optimisation of multiple objectives simultaneously ${ }^{56}$. While previously this entailed combining each of the objectives into a single unary value, algorithms such as PESA-II (Pareto envelope-based selection algorithm) select sets of conditions evenly from the Pareto front (see Fig. S5, ESI $)^{58}$. Unlike simplex optimisation, EMO is amenable to the optimisation of problems that exhibit non-linear interaction of variables. EMO has proven effective in a range of real world optimisation problems, including proving effective in maximising the experimental conditions to enhance signal and reproducibility in SERS measurements ${ }^{56}$.

Five experimental conditions were optimised: a) optical fibre core diameter, b) particle concentration, c) inner/sheath flow applied pressure ratio, d) Total applied pressure (taken to be the combined pressures applied across all 3 pumps), and e) use of a dye in the particle core stream. In order to allow direct inputting of experimental settings without the need for any calculation steps, flow rates were inputted into the genetic algorithm in the form of applied pressures across inlets. The pumps used in this study do not have integrated flow rate sensors and so flow rates are changed by changing the applied pressure. If desired however, applied pressures can be converted to flow rates by use of an adapted Poiseuille equation as given by the manufacturer. Two optical fibre core diameters were used: $50 \mu \mathrm{m}$ and $105 \mu \mathrm{m}$. A smaller fibre core diameter was expected to give a higher signal-to-noise ratio, but with a reduced diameter of light, particles in the device must be aligned into a narrow stream or they would not be counted. Higher particle concentrations have increased throughput, however very high values cause greater incidence of coincidental detection (>1 particle being detected in the interrogation zone simultaneously) and signal saturation. Inner/sheath applied pressure ratios dictate the core stream thickness as seen in Fig. $2 a$. Narrow 
core streams prevent coincidental detection but too narrow a stream prevents particle passage. Higher overall flow rates (gained from high overall applied pressures) give higher throughputs but very high values increase the particle speed beyond the data acquisition time, and can decrease the signal-to-noise ratio. A GA was used to locate an optimum balance of these conditions, to best meet the three objectives above.

An initial set of 20 runs, with varying combinations of the 5 conditions, was randomly generated by the GA (Table S1, ESI†), within the boundaries given in the Experimental section. This set formed the 1st generation of the GA. The resulting pulse spectra were analysed and ranked according to each of the 3 objectives. Resulting sets of experimental conditions and their consequent properties from the 3 generations of the GA are plotted in Fig. 3. This figure only displays the data sets without the added dye and $50 \mu \mathrm{m}$ core fibre diameters, as it was determined that these properties were not conducive to observing peaks. The presence of dye caused severe peak spectral disruption (in the form of both greatly reduced baseline light intensity, and obstruction of pulses, Fig S6), and the smaller optical fibre core size was extremely difficult to align reproducibly, preventing a reproducible counting efficiency as well as making it very difficult to gain a sufficiently high enough baseline intensity. It was apparent from the data shown in Fig. 3 that sets of conditions with low particle concentrations, low overall flow rates and low sheath/inner flow ratios failed to yield sufficient numbers of peaks to analyse, if at all (blue-coloured runs in Fig. 3c denote spectra lacking any pulses). Fig. 3 also illustrates that a region of high performance around point "A2" (see Table S1, ESI†) (denoted in Fig. 3c) was observed. The genetic algorithm appears to converge to this region quickly (Fig. 3d) after the first generation, and explores this space in subsequent generations. Notably there appears to be coherence within the data; points of high performance are proximal to points of similar performance, indicative of reproducibility within the experiment. Fig. 3e shows an example of a non-optimised particle spectra (point "I2"), with weak pulses also occurring in bursts (as opposed to periodic time increments). In contrast, Point "A2", the found optimum set of experimental conditions, produces a profile (Fig. 3f) with relatively large pulses and low variation in period. These conditions were used as a starting point for subsequent experiments evaluating particle concentration and particle size.

\section{Particle counting}

To ascertain if the pulse frequency was proportional to the concentration of the beads, a series of measurements were performed over a range of $30 \mu \mathrm{m}$ bead concentrations. The bead suspensions were run for $100 \mathrm{~s}$ at the optimum conditions set "A2" taken from Fig 3 . The resulting plot of pulse frequency versus concentration is given in Fig. 4. Pulse count increases linearly with particle concentration up to $5.5 \times 10^{4}$ particles $\mathrm{ml}^{-1}$. At concentrations higher than this the number of counted pulses plateaus. The reason the measured pulse frequency does not increase with increasing bead number past $5.5 \times 10^{4}$ particles $\mathrm{ml}^{-1}$ is attributed to the automated process for peak-calling used here. A 50-point moving average baseline light intensity was calculated across the duration of a particle run, and an intensity drop threshold of $3{ }^{*}$ SDs from this value was set for pulse calling. At higher concentration of beads, the large numbers of pulses lowers the calculated baseline intensity value, whilst increasing the intensity SD value, causing pulses to be missed. Figure S7 in the SI illustrates this effect. Using an alternative pulse-calling method could alleviate this (for example, setting a threshold for the baseline intensity calculation to eliminate pulse values being included), but for our purposes in this paper the current linear portion of the graph was adequate, and the found optimum value for $30 \mu \mathrm{m}$ diameter beads was well inside this range. Each concentration point is comprised of two averaged, separate count tests performed after full dismantling and reassembly of the device. A high correlation coefficient of 0.998 was obtained, compatible to soft-lithography techniques utilising electrochemical sensing with an expensive set-up ${ }^{62}$. 


\section{Particle sizing}

Finally, we wished to demonstrate that pulse magnitude was proportional to particle diameter. Suspensions of two sizes of polystyrene bead $\left(10 \mu \mathrm{m}, 2.5 \times 10^{5}\right.$ beads $\mathrm{ml}^{-1}$, and $30 \mu \mathrm{m}, 2.5 \times 10^{4}$ beads $\mathrm{ml}^{-1}$ ) were run at the found optimum conditions for $100 \mathrm{~s}$, followed by a mixture of the two (with concentrations of $1.25 \times 10^{4}$ beads $\mathrm{ml}^{-1}$ each). Fig. 5. Shows the pulse spectra gained from the suspensions of a) $30 \mu \mathrm{m}$ beads b) $10 \mu \mathrm{m}$ beads and c) the mixture of the two sizes, all run at the optimum conditions as defined by the GA. The pulses from the $10 \mu \mathrm{m}$ beads (an intensity of 13 counts from the baseline) have a significantly smaller magnitude than those of $30 \mu \mathrm{m}$ bead pulses (between 1-14 counts from the baseline). This smaller light intensity drop is due to the smaller bead blocking less light from the illumination fibre. Pulse magnitudes for the 3 runs were plotted as percentage signal histograms (Fig. $5 \mathrm{~d}$ ). The broad signal intensity range from the $30 \mu \mathrm{m}$ beads are likely to be due to the way the data was acquired and the movement of the particles: particles that were just entering or exiting the beam of light during data acquisition will give a lower signal than those recorded in the centre of the beam. There are two ways to improve this, the first is to use a smaller fibre optic core to ensure that the entire bead blocks the light source, however this proved difficult to get a reproducible signal in the current setup. The alternative is to increase the number of data points captured per second, ensuring that we measure the particles as it transits the light source. We can also not exclude multiple beads being present in the light at the same time, and off axial transport where the particles are at different heights in the flow device causing a variation in the signal. The bead mixture histogram had peaks that match the individual 10 and $30 \mu \mathrm{m}$ bead samples. Longer run times and raw data for the three samples can be found in Fig. S8 in the SI.

\section{Conclusions}

The first 3D printed optofluidic particle characterisation device was fabricated in approximately 5 hours, with only $\sim 1 \mathrm{hr}$ of the fabrication time spent on manual work (consisting of rinsing with isopropyl alcohol, polishing of optical fibres and application of the sealing layers). It features an integrated optical detection system that is simpler, cheaper and more user friendly than current microflow cytometer optical detection systems, as well as being able to discriminate particles by size without the need for labelling. The chip and optical fibres cost only $£ 11$ to produce, bringing the chip into the realm of disposable sensors. The experimental conditions for particle analysis were optimised via an evolutionary multiobjective optimisation algorithm. Such algorithms have proved valuable in numerous fields, including microfluidics ${ }^{63}$ and experimental optimisation ${ }^{56}$. To improve the resolution of the device to detect smaller particles, a more advanced optical system could be used, and/or a printed lens ${ }^{64}$ integrated into the microflow cytometer.

In addition, future studies may exploit the technique further to optimise the physical properties of the microfluidic device through the additive manufacturing process. This may enable the development of devices optimised for specific measurements, for instance the ability to differentiate between blood cells, or single particles and a mass of particles in an aggregation assay. Detecting smaller particles may be possible with the above system without the need for optics and lasers: the detection of particles $<10 \mu \mathrm{m}$ may be possible by increasing the signal-to-noise ratio via a smaller optical fibre core, in conjunction with a smaller channel height or 3D focusing of the particle stream.

\section{Acknowledgements}

The author would like to thank Loughborough University for the funding of this research, and David Thompson in the Wolfson School of Mechanical, Electrical and Manufacturing Engineering for his support. 


\section{Conflicts of Interest.}

The authors declare no conflicts of interest.

\section{Notes and references}

1 A. M. Urdea, L. A. Penny, S. S. Olmsted, M. Y. Giovanni, P. Kaspar, A. Shepherd, P. Wilson, C. A. Dahl, S. Buchsbaum, G. Moeller and D. C. H. Burgess, Nature, 2006, 422, 412-418.

2 J. Rantanen and J. Khinast, J. Pharm. Sci., 2015, 104, 3612-3638.

3 K. A. Koehler and T. Peters, Curr. Environ. Heal. Rep., 2015, 2, 399-411.

4 D. T. Chiu, A. J. DeMello, D. Di Carlo, P. S. Doyle, C. Hansen, R. M. Maceiczyk and R. C. R. Wootton, Chem, 2017, 2, 201-223.

5 H. Zhang, C. H. Chon, X. Pan and D. Li, Microfluid. Nanofluidics, 2009, 7, 739-749.

6 T. Konry, S. S. Bale, A. Bhushan, K. Shen, E. Seker, B. Polyak and M. Yarmush, Mikrochim Acta., 2012, 176, 251-269.

$7 \quad$ X. Wu, Y. Kang, Y. Wang, D. Xu, D. Li and D. Li, Electrophoresis, 2008, 29, 2754-2759.

8 Y. Song, H. Zhang, C. H. Chon, X. Pan and D. Li, Sensors Actuators, B Chem., 2011, 155, 930-936.

9 J. Sun, Y. Kang, E. M. Boczko and X. Jiang, Electroanalysis, 2013, 25, 1023-1028.

10 Y. Song, J. Yang, X. Pan and D. Li, Electrophoresis, 2015, 36, 495-501.

11 T. Hirono, H. Arimoto, S. Okawa and Y. Yamada, Meas. Sci. Technol., 2008, 19, 025401.

12 G. Kokkinis, S. F. Cardoso, F. A. Cardoso and I. Giouroudi, IEEE Trans. Magn., 2014, 50, 4401304.

13 J. W. Parks, M. A. Olson, J. Kim, D. Ozcelik, H. Cai, R. Carrion Jr., J. L. Patterson, R. A. Mathies, A. R. Hawkins and H. Schmidt, Biomicrofluidics, 2014, 8, 054111.

14 A. L. Givan, in Flow Cytometry Protocols, eds. T. S. Hawley and R. G. Hawley, Springer Science+Business Media, LLC, NY, 3rd edn., 2011, vol. 699, pp. 1-29.

15 H. Shapiro, Practical Flow Cytometry, John Wiley \& Sons Inc., Hoboken, NJ, 4th Ed., 2003.

16 A. Adan, G. Alizada, Y. Kiraz, Y. Baran and A. Nalbant, Crit. Rev. Biotechnol., 2017, 37, 163-176.

17 Y. Zhang, B. R. Watts, T. Guo, Z. Zhang, C. Xu and Q. Fang, Micromachines, 2016, 7, 70.

18 S. H. Cho, J. M. Godin, C.-H. Chen, W. Qiao, H. Lee and Y. H. Lo, Biomicrofluidics, 2010, 4, 043001.

19 D. Wartmann, M. Rothbauer, O. Kuten, C. Barresi, C. Visus, T. Felzmann and P. Ertl, Front. Mater., 2015, 2, 60.

20 M. I. Lapsley, L. Wang and T. J. Huang, Biomark. Med., 2013, 7, 75-78.

21 M. E. Piyasena and S. W. Graves, Lab Chip, 2014, 14, 1044-1059.

22 D. Wlodkowic and Z. Darzynkiewicz, Methods Cell Biol., 2011, 102, 105-125.

23 S. Ligler, Frances and J. S. Kim, The Microflow Cytometer, Pan Stanford Publishing, Singapore, 2010.

24 D. A. Ateya, J. S. Erickson, P. B. Howell, L. R. Hilliard, J. P. Golden and F. S. Ligler, Anal. Bioanal. Chem., 2008, 391, 1485-1498.

25 B. R. Watts, Z. Zhang, C. Q. Xu, X. Cao, and M. Lin, Electrophoresis, 2014, 35, 271-281.

26 Y. Fan, Y. Weng, and H. Sheen, Proceedings of the 12th IEEE International Conference on Nano/Micro Engineered and Molecular Systems, 2017, LA, USA, 426-429.

27 S. Etcheverry, A. Faridi, H. Ramachandraiah, T. Kumar, W. Margulis, F. Laurell and A. Russom, Sci. Rep. 2017, 7:5628.

28 K. F. Lei, in Microfluidics in Detection Science : Lab-on-a-chip Technologies, eds. F. H. Labeed and H. O. Fatoyinbo, Royal Society of Chemistry, Cambridge, 2014, pp. 1-28.

29 A. Waldbaur, H. Rapp, K. Länge and B. E. Rapp, Anal. Methods, 2011, 3, 2663-2954.

30 A. K. Au, W. Lee and A. Folch, Lab Chip, 2014, 14, 1294-1301.

31 N. Bhattacharjee, A. Urrios, S. Kang and A. Folch, Lab Chip, 2016, 16, 1720-1742.

32 A. K. Au, W. Huynh, L. F. Horowitz and A. Folch, Angew. Chemie - Int. Ed., 2016, 55, 38623881.

33 A. A. Yazdi, A. Popma, W. Wong, T. Nguyen, Y. Pan and J. Xu, Microfluid. Nanofluidics, 2016, 20, 50 . 
R. Amin, S. Knowlton, A. Hart, B. Yenilmez, F. Ghaderinezhad, S. Katebifar, M. Messina, A. Khademhosseini and S. Tasoglu, Biofabrication, 2016, 8, 022001.

35 C. M. B. Ho, S. H. Ng, K. H. H. Li and Y. Yoon, Lab Chip, 2015, 15, 3627-3637.

36 C. Chen, J. L. Erkal, B. C. Gross, S. Y. Lockwood and D. M. Spence, Anal. Chem., 2014, 86, 3240-3253.

37 P. Tseng, C. Murray, D. Kim and D. Di Carlo, Lab Chip, 2014, 14, 1491-1495.

38 P. F. O. Neill, a Ben Azouz, J. Liu, S. Marczak, Z. Slouka, H. C. Chang and D. Diamond, Biomicrofluidics, 2014, 8, 52112-1-11.

39 Y. He, Y. Wu, J. Fu, Q. Gao and J. Qiu, Electroanalysis, 2016, 28, 1658-1678.

40 C. Chen, B. T. Mehl, A. S. Munshi, A. D. Townsend, D. M. Spence and R. S. Martin, Anal. Methods, 2016, 8, 6005-6012.

41 S. Waheed, J. M. Cabot, N. P. Macdonald, T. Lewis, R. M. Guijt, B. Paull and M. C. Breadmore, Lab Chip, 2016, 16, 1993-2013.

42 A. J. Capel, S. Edmondson, S. D. R. Christie, R. D. Goodridge, R. J. Bibb and M. Thurstans, Lab Chip, 2013, 13, 4583-4590.

43 ISO / ASTM52900 - 15 Standard Terminology for Additive Manufacturing - General Principles - Terminology, https://www.astm.org/Standards/ISOASTM52900.htm, (accessed May 2017).

44 G. W. Bishop, J. E. Satterwhite-Warden, I. Bist, E. Chen and J. F. Rusling, ACS Sensors, 2016, 1, 197-202.

45 G. W. Bishop, J. E. Satterwhite, S. Bhakta, K. Kadimisetty, K. M. Gillette, E. Chen and J. F. Rusling, Anal. Chem., 2015, 87, 5437-5443.

46 J. L. Erkal, A. Selimovic, B. C. Gross, S. Y. Lockwood, E. L. Walton, S. McNamara, R. S. Martin and D. M. Spence, Lab Chip, 2014, 14, 2023-2032.

47 M. E. Snowden, P. H. King, J. A. Covington, J. V Macpherson and P. R. Unwin, Anal. Chem., 2010, 82, 3124-3131.

48 G. Gaal, M. Mendes, T. P. De Almeida, M. H. O. Piazzetta, Â. L. Gobbi, A. Riul Jr and V. Rodrigues, Sensors Actuators B Chem., 2017, 242, 35-40.

49 S. A. N. Gowers, V. F. Curto, C. A. Seneci, C. Wang, S. Anastasova, P. Vadgama, G.-Z. Yang and M. G. Boutelle, Anal. Chem., 2015, 87, 7763-7770.

50 L. Krejcova, L. Nejdl, M. A. M. Rodrigo, M. Zurek, M. Matousek, D. Hynek, O. Zitka, P. Kopel, V. Adam and R. Kizek, Biosens. Bioelectron., 2014, 54, 421-427.

51 M. Paknahad, J. S. Bachhal, A. Ahmadi and M. Hoorfar, Sensors Actuators B Chem., 2017, 241, 55-64.

52 S. Takenaga, B. Schneider, E. Erbay, M. Biselli, T. Schnitzler, M. J. Schöning and T. Wagner, Phys. Status Solidi Appl. Mater. Sci., 2015, 212, 1347-1352.

53 T. Monaghan, M. J. Harding, R. A. Harris, R. J. Friel and S. D. R. Christie, Lab Chip, 2016, 16, 3362-3373.

54 R. Walczak and K. Adamski, J. Micromech. Microeng., 2015, 25, 085013.

55 O. Okafor, A. Weilhard, J. A. Fernandes, E. Karjalainen, R. Goodridge and V. Sans, React. Chem. Eng., 2017, 2, 129-136.

56 R. M. Jarvis, W. Rowe, N. R. Yaffe, R. O'Connor, J. D. Knowles, E. W. Blanch and R. Goodacre, Anal. Bioanal. Chem., 2010, 397, 1893-1901.

57 G. Comina, A. Suska and D. Filippini, Micromachines, 2015, 6, 437-451.

58 J. Knowles, IEEE Comput. Intell. Mag., 2009, 4, 77-91.

59 A. Haciouğlu and İ. Özkol, Aircr. Eng. Aerosp. Technol., 2003, 75, 350-357.

60 C. G. Knight, M. Platt, W. Rowe, D. C. Wedge, F. Khan, P. J. R. Day, A. Mcshea, J. Knowles and D. B. Kell, Nucleic Acids Res., 2009, 37, e6.

61 J. Bongard, V. Zykov and H. Lipson, Science (80-. )., 2006, 314, 1118-1121.

62 Y. Song, H. Zhang, C. H. Chon, S. Chen, X. Pan and D. Li, Anal. Chim. Acta, 2010, 681, 82-86.

63 J. S. Kasule, J. Maddala, P. Mobed and R. Rengaswamy, Comput. Chem. Eng., 2016, 85, 94-104.

64 B. Stender, W. Mantei and R. Houbertz, Laser Tech. J., 2017, 14, 20-23. 

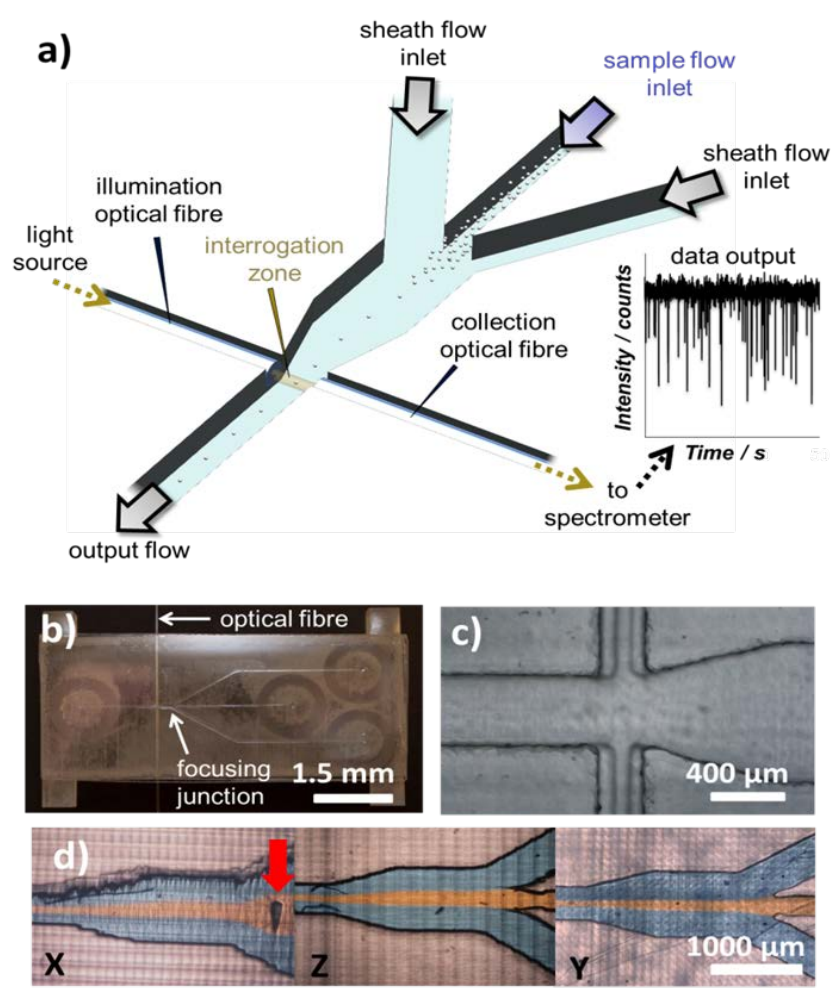

Figure 1. a) Schematic illustrating hydrodynamic focusing and detection processes in the 3D printed device. (b) Photograph of the printed device, built in Accura ${ }^{\circledR 60}$ polymer via SLA in the $Y$ build-direction (see Fig. S2, ESI + ). (c) Photograph of fibre optic junction. (d) Photographs of device focusing junctions printed in the three different build directions (left-to-right: $X, Z, Y$ ), with dye flows. 

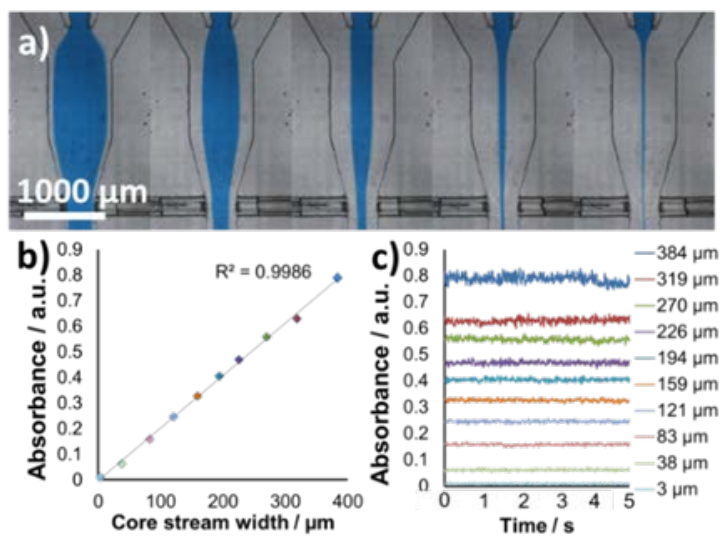

Figure 2. a) Photographs of hydrodynamic focusing of dye streams within the printed device, with inner flow applied pressure $=300 \mathrm{mbar}$, and inner/sheath applied pressure ratios (left-to-right): 2.75, 2.0, 1.0, 0.75 and 0.625. b) Plot of measured absorbance values for different dyed core stream widths, averaged over 5 s. c) Measured absorbance over time for the different dyed core stream widths. 

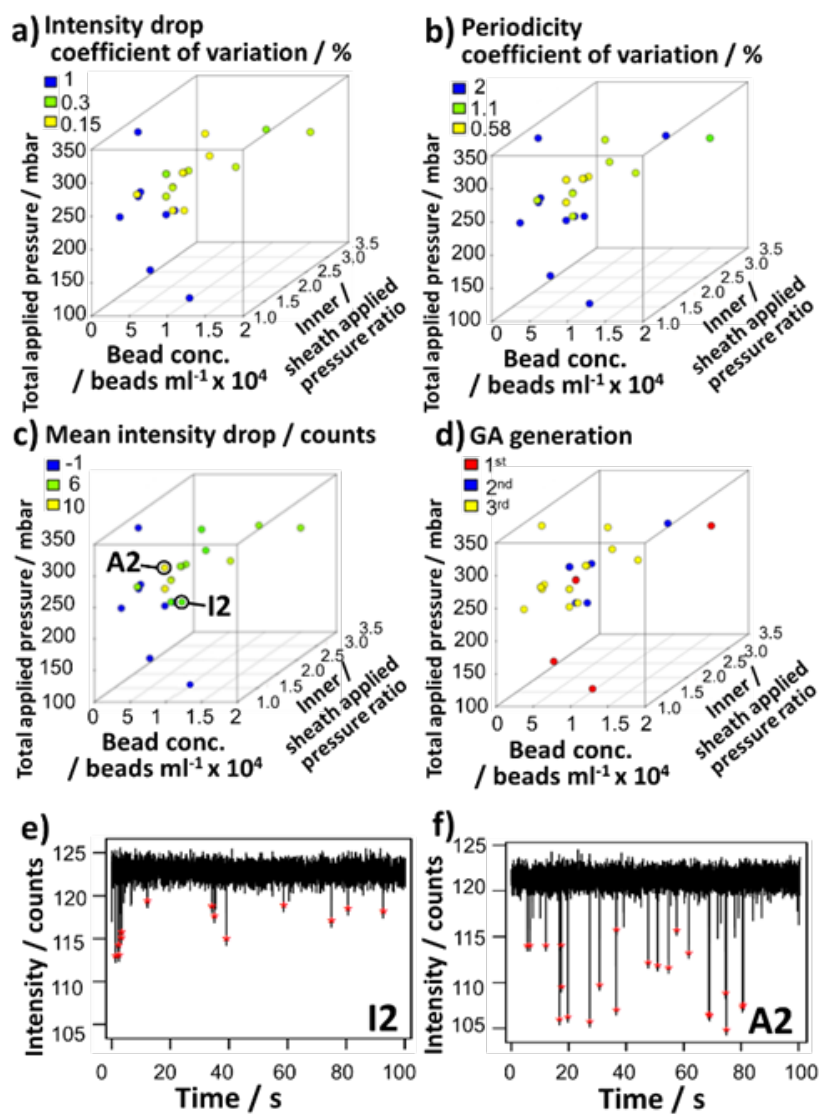

Figure 3. Optimisation of the experimental conditions required for particle analysis. Plots a)-c) mark the same evaluated experimental conditions coloured by performance in terms of a) variation in pulse magnitude, $b$ ) variation in period (time distance between peaks) and b) mean pulse magnitude (peak height). d) Displays the same points coloured by algorithm generation. (Note: conditions comprising dye or $50 \mu \mathrm{m}$ core fibre diameters are omitted from these plots, as they were less conducive to peak analysis. Consequently, there are more peaks displayed from generation 3 than generation 2, as the algorithm progressively excludes these properties.) e) Example of pulse spectrum gained under non-optimised conditions ("I2"), versus f) that gained under optimum conditions "A2" (see Table S1, ESIT). 


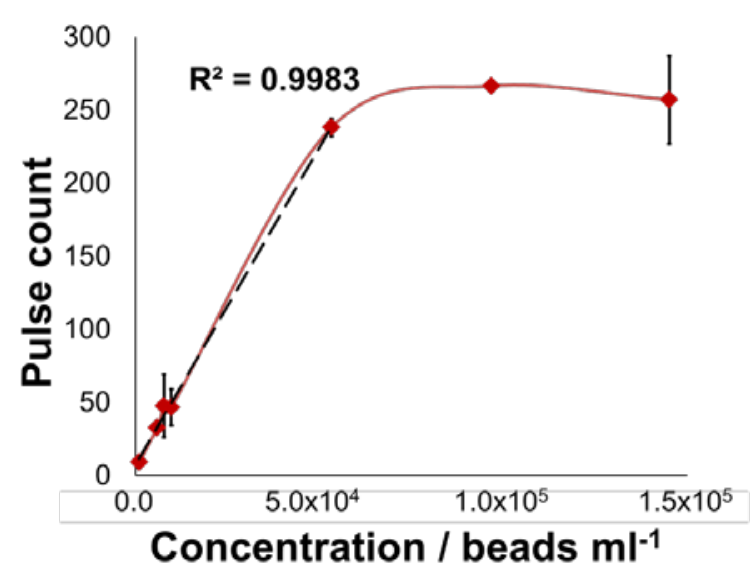

Figure 4. Plot of pulse count versus particle concentration over 100s, done at optimised conditions for $30 \mu \mathrm{m}$ d. beads 

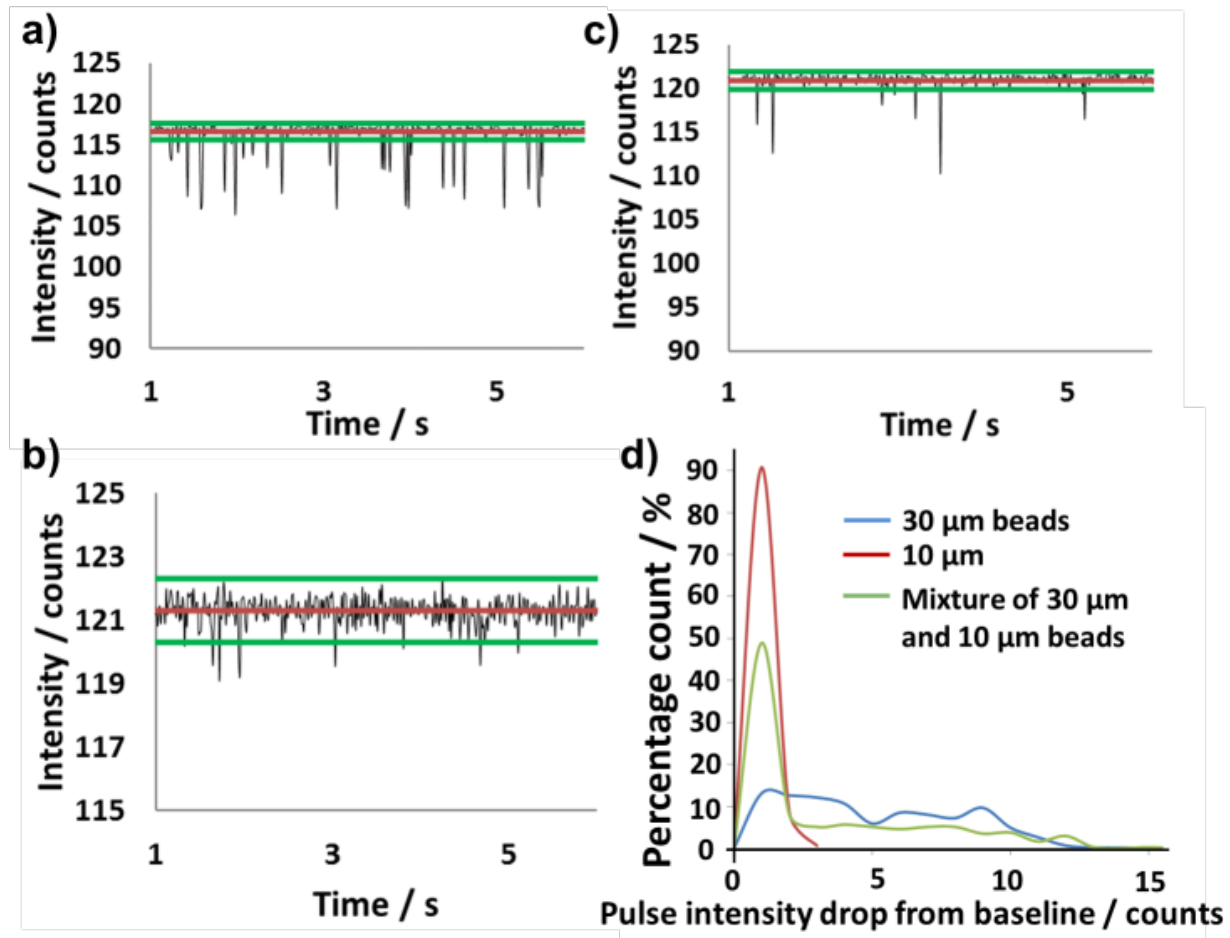

Figure 5. Pulse spectra collected at found optimised conditions for a) $30 \mu \mathrm{m}$ diameter beads, $2.5 \mathrm{x}$ $10^{4}$ beads $\mathrm{ml}^{-1} \mathrm{~b}$ ) $10 \mu \mathrm{m}$ diameter beads, $2.5 \times 10^{4}$ beads $\mathrm{ml}^{-1} \mathrm{c}$ ) a 1:1 mixture of the two size beads, each $1.25 \times 10^{4}$ beads $\mathrm{ml}^{-1}$. The red line represents the average baseline light intensity value, with the green lines representing $3 \times S D$ of the noise from a blank run. d) Percentage count versus signal intensity for $10 \mu \mathrm{m}$ diameter beads $\left(2.5 \times 10^{4}\right.$ beads $\left.\mathrm{ml}^{-1}\right), 30 \mu \mathrm{m}$ diameter beads $\left(2.5 \times 10^{4}\right.$ beads $\mathrm{ml}-1)$, and the 1:1 mixture $\left(1.25 \times 10^{4}\right.$ beads $\left.\mathrm{ml}^{-1}\right)$. 